\title{
Operational criteria for the classification of chronic alcoholics: identification of Wernicke's encephalopathy
}

\author{
D Caine, G M Halliday, J J Kril, C G Harper
}

Neuropsychology Unit, Royal Prince Alfred Hospital,

Camperdown, 2050,

Australia

D Caine

Prince of Wales

Medical Research

Institute, High Street,

Randwick, 2031,

Australia

G M Halliday

Neuropathology

Division, Departments

of Pathology,

University of Sydney,

2006 and Royal Prince

Alfred Hospital,

Camperdown, 2050,

Australia

G M Halliday

J J Kril

C G Harper

Correspondence to:

Dr G Halliday, Prince of

Wales Medical Research

Institute, Villa 2, High

Street, Randwick. 2031,

Australia.

Received 25 March 1996

and in final revised form

30 August 1996

Accepted 2 September 1996

\begin{abstract}
Objectives-To establish better operational criteria for the diagnosis of Wernicke's encephalopathy. Current criteria for diagnosing Wernicke's encephalopathy require the presence of three clinical signs (oculomotor abnormalities, cerebellar dysfunction, and an altered mental state), although it has often been reported that most patients do not fulfil all these criteria.
\end{abstract}

Methods-The clinical histories of 28 alcoholics with neurological and neuropsychological assessments and definitive neuropathological diagnoses were examined to determine clinical signs for use in a screening schedule. Operational criteria were then proposed for differentiating patients with Wernicke's encephalopathy alone or in combination with Korsakoff's psychosis or hepatic encephalopathy. The new criteria for Wernicke's encephalopathy require two of the following four signs; (1) dietary deficiencies, (2) oculomotor abnormalities, (3) cerebellar dysfunction, and (4) either an altered mental state or mild memory impairment. Reproducibility and validity testing of these criteria were performed on 106 alcoholics screened from a large necropsy sample.

Results-Despite rater variability with regard to specific symptoms, within and between rater reliability for diagnostic classification using the criteria retrospectively on patient records was $100 \%$ for three independent raters. Validity testing showed that Wernicke's encephalopathy was underrecognised only when occurring with hepatic encephalopathy $(50 \%$ sensitivity).

Conclusions-By contrast with current criteria, the proposed operational criteria show that the antemortem identification of Wernicke's encephalopathy can be achieved with a high degree of specificity.

$(\mathcal{F}$ Neurol Neurosurg Psychiatry 1997;62:51-60)

Keywords: alcoholism; diagnostic criteria; neurological impairment; Wernicke-Korsakoff syndrome

It is well known that the pathology of Wernicke's encephalopathy may not be associated with the full clinical triad and that in the acute phase clinical symptoms may resolve with thiamine treatment. ${ }^{1-11}$ These findings suggest that there can be a complete resolution of the underlying brain abnormality in Wernicke's encephalopathy, similar to the resolution of neuropathology in hepatic encephalopathy. However, unlike hepatic encephalopathy, in which the clinical signs are a precursor of the pathology, many patients with the neuropathology of Wernicke's encephalopathy do not have recorded signs of the classic triad. ${ }^{12}$ This raises the question of whether the clinical correlates of the pathological lesions are being missed during life or whether the pathology represents clinically silent lesions. A similar situation is seen in Alzheimer's disease, in which many nondemented aged patients show a similar type and distribution of neuropathology to their demented counterparts. ${ }^{12}$ Recently research into the pathophysiology of aging and Alzheimer's disease has been successfully advanced by the use of operational criteria which can be applied across clinics and laboratories. ${ }^{1314}$ The aim of the present study is to propose operational criteria for the diagnosis of Wernicke's encephalopathy.

Most patients world wide with Wernicke's encephalopathy are alcoholics, ${ }^{15}$ with $30 \%-$ $80 \%$ of chronic alcoholics having the clinical or biochemical signs of thiamine deficiency. ${ }^{16}$ Two extensive clinicopathological studies ${ }^{1}{ }^{2}$ found that the incidence of oculomotor abnormalities was low and therefore reliance on the classic triad would result in significant underrecognition of these patients during life. Because an altered mental state is also a common neurological sign associated with hepatic encephalopathy, ${ }^{17}$ the overlap between the two diseases poses substantial and often unrecognised difficulties for diagnosis. Operational criteria for recognising and separating these common conditions are needed. Therefore the aim of the present study was to devise and test such operational criteria for classifying chronic alcoholics for both treatment and research. We have assessed only alcoholics in order to analyse a large and relatively homogeneous group of patients with Wernicke's encephalopathy. The criteria account for the diagnoses of other common medical complications associated with this patient population. ${ }^{4}$ Key signs for operational criteria for diagnosis are proposed and the reliability and predictive validity (specificity and sensitivity) of using these criteria to classify chronic alcoholics tested.

Methods

To determine characteristic signs and 
symptoms for alcoholics with and without Wernicke's encephalopathy, the histories of 28 patients, well known to the neuropsychology unit, Royal Prince Alfred Hospital, who had died between 1989 and 1993 and had necropsy confirmation of their clinical diagnoses were examined. These had a variety of neurological presentations, including eight patients with Wernicke's encephalopathy (classic triad but with no evidence of a permanent amnesic syndrome), six patients with the Wernicke-Korsakoff syndrome (alcoholics with Wernicke's encephalopathy fulfilling current criteria for Korsakoff's psychosis ${ }^{18-20}$ ), nine patients with hepatic encephalopathy (neurological abnormalities associated with decompensated liver disease ${ }^{17}$ ), and eight patients with alcohol related brain dysfunction (neurological or neuropsychological impairment, although not fulfilling the criteria for Wernicke's encephalopathy, WernickeKorsakoff syndrome, or hepatic encephalopathy). Three patients had both Wernicke's encephalopathy and hepatic encephalopathy. In all patients the clinical diagnosis matched the neuropathological diagnosis, patients with alcohol related brain dysfunction having no neuropathological abnormality.

STANDARDISED CLINICAL EXAMINATION

Patients in the neuropsychology unit underwent a 90 minute clinical assessment by a consultant neurologist or neurology registrar, comprising a detailed history following a standard protocol, a complete general medical examination, and a detailed neurological examination including an examination of mental status. They also underwent clinical neuropsychological assessment which included

Table 1 Schedule of clinical signs and symptoms with domains highlighted (see text for definitions)

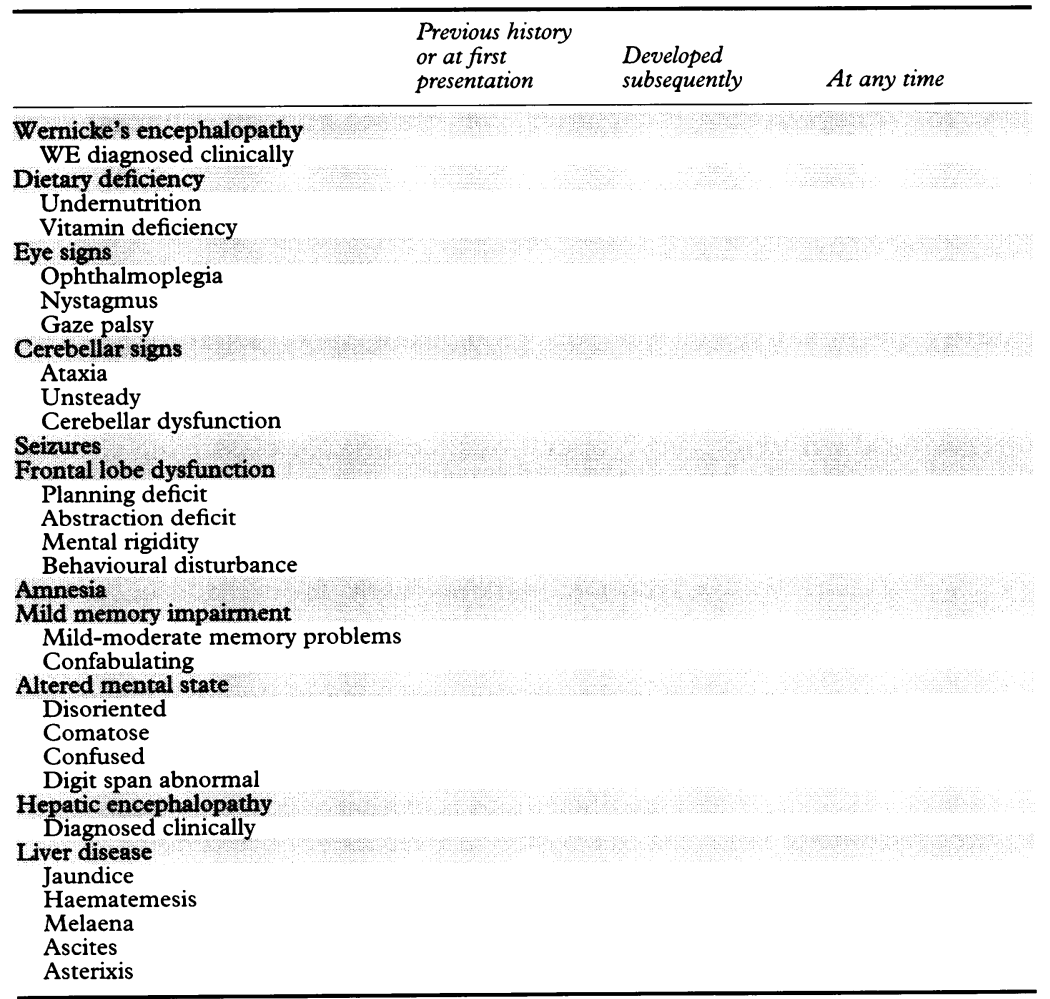

standardised administration and scoring of the following neuropsychological tests: Wechsler memory scale, Rey auditory verbal learning test, Rey-Osterrieth complex figure test, trail making test, and Stroop test. ${ }^{21}$ The diagnosis of Wernicke's encephalopathy, WernickeKorsakoff syndrome, hepatic encephalopathy, or alcohol related brain dysfunction was made using the history obtained, information from referring agencies, the results of the clinical and neuropsychological examinations, and those of any additional tests which may have been ordered.

\section{PATHOLOGICAL DIAGNOSIS}

A full necropsy was performed for all patients including macroscopic and microscopical examination of all organs. A detailed neuropathological examination was performed. The external brain features were examined before and after fixation in neutral buffered formalin for 14 days. The brain was then embedded in 3\% agar and sectioned at $3 \mathrm{~mm}$ intervals in the coronal plane with a rotary slicer. The cut surfaces of each slice were examined for neuropathological abnormalities. Standardised blocks of the superior frontal, superior parietal, inferior temporal, and parahippocampal cortices, hippocampus, amygdala, anterior and posterior basal ganglia including the basal forebrain, thalamus, mamillary bodies of the hypothalamus, midbrain, pons, medulla oblongata, and cerebellum (vermis, lateral cortices and dentate nuclei) were sampled for paraffin embedding. Sections were cut at $10 \mathrm{~mm}$ and stained with haematoxylin and eosin, luxol fast blue, and silver stains before microscopical examination and diagnosis (by $\mathrm{CGH}$ ). Other specialised stains were performed if required to exclude cerebral infarction, head injury, or degenerative conditions such as Alzheimer's disease. Serious liver disease was diagnosed if liver cirrhosis or hepatitis was confirmed at necropsy. Hepatic encephalopathy was diagnosed if the patient had serious liver disease and Alzheimer type II astrocytes in the brain (common in the basal ganglia, cerebral cortex, or pons). Wernicke's encephalopathy was diagnosed when mamillary body and periventricular lesions were evident. ${ }^{22}{ }^{23}$ In acute Wernicke's encephalopathy the pathology was largely confined to abnormalities of blood vessels with petechial haemorrhages commonly occurring. In chronic Wernicke's encephalopathy neuronal loss and gliosis were evident in the mamillary bodies. A diagnosis of normal brain was made if no neuropathological abnormality was detected.

\section{EVALUATION OF CLINICAL SIGNS AND SYMPTOMS}

A range of clinical signs and symptoms representing deficits in the major clinical domains known to be affected in alcoholics ${ }^{4}$ was evaluated for establishing operational criteria. Related clinical symptoms and alternative terms used to describe a sign or symptom were grouped together under each of these domains and a standardised schedule of the clinical 
signs designed (table 1). For example, cerebellar damage resulting in ataxia is commonly present in alcoholics, but in the absence of formal testing it is often recorded as an unsteadiness. Therefore both ataxia and unsteadiness were used as indicators of cerebellar dysfunction. The presence of either of these signs was sufficient to result in a positive score for cerebellar abnormalities.

Evaluation of the clinical profiles of the 28 patients disclosed 11 commonly encountered clinical domains. These were Wernicke's encephalopathy (diagnosed during life using the classic triad), dietary deficiencies (a body mass index lower than 2 SD below normal as evidence of undernutrition, a history of grossly impaired dietary intake, or an abnormal thiamine status), eye signs (oculomotor abnormalities such as ophthalmoplegia, nystagmus, or gaze palsy), cerebellar signs (see above, or abnormalities of past pointing, dysdiadokokinesia, or impaired heel-shin testing), seizures (either as part of a withdrawal syndrome or in isolation, or a longstanding history of anticonvulsant medication), frontal lobe dysfunction (abnormalities in planning, insight, or abstraction with formal neuropsychological testing or when neurological examination elicited these characteristics), amnesia (a stable and persisting inability to form new memories), mild memory impairment (failure to remember two or more words in the four item memory test, ${ }^{24}$ or impairment on more elaborate neuropsychological tests of memory function), altered mental state (disorientation in two of three fields, confused, an abnormal digit span, or comatose); hepatic encephalopathy (grades 3 or 4 , in which signs of impaired mental state range from confusion to coma ${ }^{17}$ ), and liver disease (signs of systemic abnormalities secondary to chronic liver disease including melaena, haematemesis, jaundice, ascites, or asterixis). Abnormal liver function tests in isolation were not considered sufficient to have a positive score for liver disease. In addition, poor performance on memory tests on only one occasion was only considered sufficient for a positive score for amnesia if the patient was unable to form any new memories.

ESTABLISHING OPERATIONAL CRITERIA

Retrospective patient note analysis was con-

Table 2 Operational criteria for the clinical diagnosis of chronic alcoholics. See text for definition of clinical signs. Multiple diagnoses are possible

\begin{tabular}{|c|c|c|c|c|c|c|}
\hline \multirow[b]{2}{*}{ Clinical domains } & \multicolumn{6}{|c|}{ Clinical diagnoses } \\
\hline & $\begin{array}{l}\text { No clinical } \\
\text { signs }\end{array}$ & $\begin{array}{l}\text { Liver disease } \\
\text { only }\end{array}$ & $A R B D$ & $H E$ & $W E$ & $W K S$ \\
\hline $\begin{array}{l}\text { WE } \\
\text { Dietary deficiency } \\
\text { Eye signs } \\
\text { Cerebellar signs } \\
\text { Seizures } \\
\text { Frontal lobe } \\
\text { dysfunction }\end{array}$ & $\begin{array}{l}\text { Absent } \\
\text { Absent } \\
\text { Absent } \\
\text { Absent } \\
\text { Absent }\end{array}$ & $\begin{array}{l}\text { Absent } \\
\text { Absent } \\
\text { Absent } \\
\text { Absent } \\
\text { Absent }\end{array}$ & $\begin{array}{l}\text { Absent } \\
\star \\
\star \\
\star\end{array}$ & Absent & $\begin{array}{l}\text { Optional } \\
t \\
t \\
t\end{array}$ & $\begin{array}{l}\text { Optional } \\
t \\
t \\
t\end{array}$ \\
\hline $\begin{array}{l}\text { dysfunction } \\
\text { Amnesia } \\
\text { Mild memory }\end{array}$ & $\begin{array}{l}\text { Absent } \\
\text { Absent }\end{array}$ & $\begin{array}{l}\text { Absent } \\
\text { Absent }\end{array}$ & * & Absent & Absent & Present \\
\hline $\begin{array}{l}\text { impairment } \\
\text { Altered mental state } \\
\text { HE } \\
\text { Liver disease }\end{array}$ & $\begin{array}{l}\text { Absent } \\
\text { Absent } \\
\text { Absent } \\
\text { Absent }\end{array}$ & $\begin{array}{l}\text { Absent } \\
\text { Absent } \\
\text { Absent } \\
\text { Present }\end{array}$ & $\begin{array}{l}\star \\
\text { Absent } \\
\text { Absent }\end{array}$ & $\begin{array}{l}\text { Present } \\
\text { Optional } \\
\text { Present }\end{array}$ & $\begin{array}{l}+\bullet \\
\text { t. } \\
\text { Absent }\end{array}$ & $\begin{array}{l}\text { t. } \\
\text { Á } \\
\text { Absent }\end{array}$ \\
\hline
\end{tabular}

*Any one of these signs; tany two of these signs, but not both of those marked -

ARBD = alcohol related brain dysfunction; HE = hepatic encephalopathy; WE $=$ Wernicke's encephalopathy; WKS = Wernicke-Korsakoff syndrome. ducted blind to the neuropathological diagnosis using the standardised schedule to record the presence or absence of clinical features at two time points: symptoms present at or before initial neurological examination and symptoms occurring after initial presentation. The clinical domains were then categorised according to pathological diagnosis. Operational criteria for different diagnoses were devised on the basis of this information (table 2).

\section{VALIDATING THE OPERATIONAL CRITERIA \\ Test population}

All adult patients necropsied between 1986 and 1994 from the Royal Prince Alfred Hospital, as well as $10 \%$ of necropsied patients from the New South Wales Institute of Forensic Medicine (referred for neuropathological examination), constituted the base from which the test samples were drawn (4600 patients). Of these 303 had a history of alcoholism ( $7 \%$ of sample) based on information from telephone interviews to general practitioners, written questionnaires to relatives of patients, and clinical details from hospital records. Alcoholism was established if greater than $80 \mathrm{~g}$ ethanol was consumed each day for most of their adult life (usually $>30$ years) as recorded from these multiple sources. Informant histories have been shown to provide accurate assessment of alcohol intake. ${ }^{25}$ Exclusion criteria were an average daily alcohol consumption of less than $80 \mathrm{~g}$ ethanol, inadequate clinical history (no neurological examination or single admission only), or neuropathological evidence of a cerebral infarction, head injury or neurodegenerative condition (for example, Alzheimer's disease). A total of 197 of the 303 alcoholics selected were excluded, leaving 106 alcoholics for the study (35\% of alcoholics, $2 \cdot 3 \%$ of the total sample, 16 women and 90 men, including the 28 patients used for establishing the operational criteria). The age range was 33 to 79 years (mean 58 (SD 10)).

\section{INCLUSION CRITERIA AND DEFINITION OF PATIENTS}

Inclusion criteria were consumption of greater than $80 \mathrm{~g}$ ethanol per day for most of their adult life, neurological assessment, clinical assessment at more than one time point, full necropsy, interview with general practitioner or relatives, and an extensive and systematic neuropathological examination that excluded conditions other than those directly attributable to chronic alcohol consumption.

\section{TESTING THE OPERATIONAL CRITERIA}

The standardised schedule and operational criteria were applied to the longitudinal patient records of all 106 patients for clinical patient classification. About $30 \%$ of each patient type were randomly selected for analyses of between and within rater reliability ( 32 patients in total). These patients were classified independently by three researchers blind to neuropathological diagnosis (DC, GMH, JJK). Symptoms and signs occurring at any stage were used for classification. The predic- 
tive validity of the clinical classification against the criterion of clinicopathological diagnosis is expressed in terms of sensitivity and specificity ${ }^{26}$ where sensitivity $=$ (true positives $/$ (true positives + false negatives) $) \times 100 \%$ and specificity $=$ (true negatives $/$ (false positives + true negatives) $) \times 100 \%$. The microscopical pathology for all patients with either a false clinical but positive pathological diagnosis or a true clinical but negative pathological diagnosis $(17 \%$ of all patients) was carefully rechecked blind to the clinical classification (by $\mathrm{CGH}$ ). If discrepencies between diagnoses still existed (10 patients), the neuropathology was rechecked by two independent researchers (GMH, JJK) and neuropathological diagnosis determined by consensus agreement of all three researchers. The original neuropathological diagnosis was confirmed in all of these patients.

\section{Results}

DIAGNOSTIC GROUPS

Application of the operational criteria to the 106 patients confirmed the major diagnoses identified in the 28 detailed patients and established a pattern for six different diagnostic groups. The clinical characteristics and progression of symptoms for each group follow.

Alcoholics with compensated liver disease only had clinical evidence of compensated liver disease in concert with the pathology for serious liver disease and a normal brain. patients were excluded if neurological impairment or dietary deficiency was evident. These patients had one or more of the symptoms of liver disease, but did not progress to develop all symptoms or hepatic coma.

Alcoholics with hepatic encephalopathy had clinical evidence of decompensated liver disease (altered mental state) in concert with the pathology for serious liver disease and hepatic encephalopathy. Patients were excluded if another encephalopathy was diagnosed clinically or if dietary deficiencies, eye signs, cerebellar signs, mild memory impairment, or amnesia were evident, unless the patient had also been given a definitive clinical diagnosis of hepatic encephalopathy.

The presence of cirrhosis of the liver was often not apparent until the patient presented with symptoms of decompensated liver disease. Therefore these patients represent the more severe end of the range of patients with liver disease. The most frequent symptoms included the combination of jaundice, ascites, melaena, and haematemesis, whereas asterixis was less frequent (patients 1 and 2, appendix). The longest interval between initial presentation with liver disease and death was 15 months (patient 2) and was independent of whether the patient ceased drinking during this time. Therefore, although these patients may have had cirrhosis of the liver for an extended period, mortality seemed inevitable after the development of hepatic encephalopathy and was most commonly due to sepsis, gastrointestinal haemorrhage, or raised intracranial pressure.

Alcoholics with Wernicke's encephalopathy alone had a definitive clinical diagnosis of Wernicke's encephalopathy (classic triad) or clinical evidence of at least two of the four following signs; (1) dietary deficiencies, (2) eye signs, (3) cerebellar signs, and (4) either an altered mental state or mild memory impairment; and Wernicke's encephalopathy at necropsy. Amnesic patients and patients with a diagnosis of hepatic encephalopathy were excluded from this group. All patients had a progressive and usually short clinical course (average of three years between first presentation and death). At least two of the four signs of Wernicke's encephalopathy were present at, or soon after, first presentation (patients 3 and 4, appendix). In many patients only a single sign of the Wernicke's encephalopathy triad was noted initially, whereas at subsequent presentations there were usually additional neurological signs. Most were still drinking at the time of death. It should be noted that clinically, many of these patients did not fulfill current diagnostic criteria using the full clinical triad (eye signs, cerebellar signs, and an altered mental state). We found that patients with Wernicke's encephalopathy pathology had a minimum of two clinical signs which may have included dietary deficiencies. Thus some patients were included with only one neurological abnormality in the presence of dietary deficiencies. This criterion differs substantially from the classic definition.

Alcoholics with the Wernicke-Korsakoff syndrome fulfilled the above criteria for the diagnosis of Wernicke's encephalopathy and had documented evidence of amnesia and disorientation in the absence of an acute confusional state (patients 5 and 6, appendix). These patients were often institutionalised due to their profound memory dysfunction and therefore tended to have a longer history of neurological signs and symptoms ( $>10$ years) documented either in patient notes or inferred from nursing home institutionalisation. Initial presentation was usually associated with the effects of intoxication or withdrawal (including seizures). There was progressive deterioration in both the range of neurological symptoms and the severity of cognitive symptoms (patient 6). In particular, stable amnesia was usually noted after detoxification at either the initial or second presentation (within one year). At this time institutionalisation usually occurred and was associated with a reduction or cessation of drinking and improved nutritional status. This is likely to contribute to the relatively long survival time of these patients.

Alcoholics with alcohol related brain dysfunction had clinical evidence of neurological impairment in at least one domain (cerebellar signs, seizures, frontal lobe dysfunction, memory impairment, altered mental state), but did not reach clinical criteria for any of the diagnostic groups above (patients 7 and 8, appendix). These patients had no significant neuropathology and usually presented with the full range of withdrawal symptoms (including seizures, patient 7) or with complex cognitive deficits (dissimilar to the Wernicke-Korsakoff syndrome in that memory impairment was 
either mild (patient 8), or, if amnesic, associated with a longstanding history of seizures). Neither eye signs nor dietary deficiencies were ever seen in these patients (patients 7 and 8). Most patients had longstanding histories of seizures (most had epilepsy diagnosed in early adulthood) or neuropsychiatric abnormalities with significant frontal lobe features (mostly coincident with their history of alcoholism).

Alcoholics without neurological complications were defined by exclusion criteria. Patients were excluded if they fulfilled any of the criteria above or if dietary deficiencies, liver disease, or neurological, psychological, or neuropathological abnormalities were evident. These alcoholic patients usually presented with intoxication, unsteadiness, blackouts, or withdrawal symptoms but without any permanent sequelae; in particular, no neurological signs or cognitive dysfunction were evident (patient 9, appendix).

Alcoholics with multiple diagnoses were also found. Some patients with neurological impairment also had evidence of liver disease. Compensated liver disease did not contribute to the neurological signs in any patient. However, significant overlap in clinical signs was evident in patients with hepatic encephalopathy and Wernicke's encephalopathy. In all such patients, the course of hepatic encephalopathy followed that described above for hepatic encephalopathy alone, although it was somewhat more prolonged. In patients with Wernicke's encephalopathy and hepatic encephalopathy diagnosed during life, the presentation of Wernicke's encephalopathy and hepatic encephalopathy occurred concurrently (patient 10, appendix), possibly indicating a more classic presentation of the Wernicke's encephalopathy triad in these patients. One patient had a history of the Wernicke's encephalopathy triad and Korsakoff's psychosis which preceded the complication of hepatic encephalopathy (patient 11, appendix).

\section{RELIABILITY AND PREDICTIVE VALIDITY OF OPERATIONAL CRITERIA}

In the $30 \%$ of patients randomly chosen for reanalysis of patient classification, rater retesting gave the same diagnosis in all patients ( $100 \%$ accuracy) even with test-retest intervals of up to four months. Further, despite between rater agreement for individual signs and symptoms on the standardised schedule of only $83 \%$, it was $100 \%$ for diagnostic classification in all instances. This indicates that grouping symptoms and signs into broad domains for patient classification is reliable and reproducible with regard to overall diagnosis while allowing for interrater differences with regard to specific signs and symptoms.

Table 3 shows the sensitivity of the operational criteria and the percentage of patients with different clinical signs in each alcoholic group. Only the diagnosis of Wernicke's encephalopathy in combination with amnesia or hepatic encephalopathy proved problematic for retrospective classification. Out of 40 patients with Wernicke's encephalopathy pathology, six were not clinically diagnosed using the operational criteria, whereas only nine patients fulfilled the classic clinical triad $(22.5 \%)$. Using the operational criteria, two patients with Korsakoff's psychosis and a history of alcoholism were institutionalised for an extended time and had no clinical record of the signs of Wernicke's encephalopathy. The remaining patients had additional hepatic encephalopathy clinically and pathologically, and the clinical signs of Wernicke's encephalopathy may have been less obvious.

Altered mental state was found at some stage in all encephalopathic patients and mild memory impairment was seen in most groups, but particularly those classified with alcohol related brain dysfunction or Wernicke's encephalopathy (either alone or in combination; table 3). Seizures were most prevalent in patients with alcohol related brain dysfunction, although the Wernicke-Korsakoff syndrome group also had an above average number of such patients (table 3). Of particular importance is the finding that cerebellar signs concentrated in all patients classified with Wernicke's encephalopathy, although patients with the Wernicke-Korsakoff syndrome had eye signs more often than those with Wernicke's encephalopathy or Wernicke's encephalopathy and hepatic encephalopathy (table 3). Conversely, there was a high prevalence of dietary deficiencies in patients with Wernicke's encephalopathy and Wernicke's encephalopathy plus hepatic encephalopathy compared with the Wernicke-Korsakoff syndrome. This is most likely due to the institutionalisation of many in this group. Other signs concentrated in particular alcoholic groups because of the inclusion/exclusion criteria. Dietary deficiencies and eye signs occurred only in partients with Wernicke's encephalopathy (with or without amnesia or hepatic encephalopathy), amnesia was most common in patients with the WernickeKorsakoff syndrome, and liver disease and hepatic encephalopathy were common in patients with these pathological diagnoses (table 3).

Table 3 also shows the specificity of the operational criteria and the percentage of overlapping clinical signs in each alcoholic group. The false positive rate for clinical classification can be obtained by subtracting the specificity value from 100 . Interestingly, only in patients classified with hepatic encephalopathy or liver disease could the diagnosis not be substantiated on pathological grounds. Of the 18 patients classified with hepatic encephalopathy using the operational criteria, no Alzheimer type II astrocytes could be found in the brains of two patients. Both patients had serious liver pathology, and examination of the clinical records showed evidence of decompensated liver disease. Yet despite this, hepatic encephalopathy could not be confirmed neuropathologically, although death occurred without longstanding coma in both patients (within three hours of coma onset). Of the 11 patients fulfilling the operational criteria for compensated liver disease only, four had no 
Table 3 Specificity and sensitivity of the clinical diagnosis based on pathological criteria and the proportion of patients with different clinical signs. Clinical signs which concentrate in particular alcoholic groups are in bold

\begin{tabular}{|c|c|c|c|c|c|c|c|c|}
\hline & \multicolumn{8}{|c|}{ Clinical diagnoses } \\
\hline & $\begin{array}{l}\text { All } 106 \\
\text { patients }\end{array}$ & $\begin{array}{l}\text { No clinical } \\
\text { signs } \\
(n=22)\end{array}$ & $\begin{array}{l}\text { Liver disease } \\
\text { only } \\
(n=11)\end{array}$ & $\begin{array}{l}A R B D \\
(n=15)\end{array}$ & $\begin{array}{l}H E \\
(n=18)\end{array}$ & $\begin{array}{l}W E \\
(n=16)\end{array}$ & $\begin{array}{l}\text { WKS } \\
(n=16)\end{array}$ & $\begin{array}{l}W E+H E \\
(n=8)\end{array}$ \\
\hline Sensitivity & 94 & 100 & 100 & 100 & 100 & 100 & 88 & 50 \\
\hline Specificity & 99 & 100 & 96 & 100 & 98 & 100 & 100 & 100 \\
\hline Mean number of signs $(/ 11)$ & 2 & 0 & 1 & 2 & 3 & 3 & $\cdot 5$ & $\cdot 5$ \\
\hline \multicolumn{9}{|l|}{ Clinical domains: } \\
\hline WE & 8 & 0 & 0 & 0 & 0 & $\cdot 31$ & 13 & $\cdot 25$ \\
\hline Dietary deficiency & 9 & 0 & 0 & 0 & 0 & $\cdot 25$ & 13 & $\cdot 38$ \\
\hline Eye signs & 19 & 0 & 0 & 0 & 0 & 31 & .56 & 25 \\
\hline Cerebellar signs & 36 & 0 & 0 & 36 & 17 & .81 & .81 & 50 \\
\hline Epilepsy & 16 & 0 & 0 & .50 & 11 & 19 & $\cdot 32$ & 0 \\
\hline Frontal lobe dysfunction & 14 & 0 & 0 & 14 & 0 & .31 & .44 & 13 \\
\hline Amnesia & 17 & 0 & 0 & 14 & 0 & 0 & $\cdot 100$ & 0 \\
\hline Mild memory impairment & 29 & 0 & 0 & 50 & 6 & .63 & 50 & $\mathbf{5 0}$ \\
\hline Altered mental state & 29 & 0 & 0 & 0 & 56 & 31 & 56 & .63 \\
\hline $\mathrm{HE}$ & 22 & 0 & 0 & 0 & $\bullet 89$ & 0 & 0 & .88 \\
\hline Liver disease & 47 & 0 & $\cdot 100$ & 21 & $\cdot 100$ & 44 & 31 & 75 \\
\hline
\end{tabular}

For abbreviations see table 2. • > Twice the proportion seen in all 106 patients. Values apart from mean number are $\%$.

serious liver pathology. Seven of 16 patients classified with Wernicke's encephalopathy also fulfilled the criteria for liver disease, although three of these patients had no serious liver pathology. All seven patients with a false positive diagnosis for liver disease using the operational criteria had clinical symptoms and signs that could be attributed to concomitant gastrointestinal disease (for example, gastric ulcer) producing melaena or haematemesis.

\section{Discussion}

The operational criteria reported here can be used to classify accurately alcoholics with neurological deficits. The criteria were designed to differentiate alcoholics with and without the Wernicke-Korsakoff syndrome, and to differentiate Wernicke's encephalopathy from hepatic encephalopathy as these represent longstanding diagnostic difficulties in alcohol research. ${ }^{1728-31}$ Other medical complications often encountered in alcoholics, but which do not result in CNS deficits, were not incorporated. By grouping together signs and symptoms into clinical domains and redefining current diagnostic criteria for Wernicke's encephalopathy, the overall accuracy of the proposed criteria is high and the need for point to point agreement on individual signs is minimised.

The existing clinical triad for Wernicke's encephalopathy was modified so that the diagnosis only required any two of the following signs; eye signs, cerebellar dysfunction, altered mental state, and dietary deficiencies. This modification produced highly specific and non-overlapping diagnoses for Wernicke's encephalopathy, the Wernicke-Korsakoff syndrome, hepatic encephalopathy, and Wernicke's encephalopathy plus hepatic encephalopathy $\quad(98 \%-100 \%$ specificity $)$. Furthermore, sensitivity for the diagnosis of Wernicke's encephalopathy was improved from $31 \%$ using the classic triad to $100 \%$ with the proposed criteria. The use of the proposed operational criteria for the classification of neurological impairment in chronic alcoholics will allow Wernicke's encephalopathy to be diagnosed with a high degree of confidence.
The validation of the criteria relies on adequate testing procedures. The test population studied was derived from both hospital and forensic sources, representing a broader cross section of patients than those from a single source. The use of forensic material minimised the bias of patient selection towards those with overt neurological complications, as all sudden or accidental deaths are subject to forensic necropsy in Australia. In addition, patients with a wide variety of neurological diseases were initially selected for neuropathological evaluation and from these, alcoholics were chosen for study. Pathological diagnoses were revalidated for all patients that were clinically mismatched. Because of the retrospective analysis and inclusion criteria, a proportion of patients at the lower end of hazardous alcohol consumption may not have been included in our alcoholic population. However, these patients are least likely to have medical complications due to their alcoholism and may only have neurological deficits disclosed by detailed neuropsychological assessment. ${ }^{32} \mathrm{We}$ are therefore confident of the validity of our sample and test populations and that our methodology has not produced substantial bias towards alcoholics with neurological impairment.

\section{DIFFERENTIATION OF DIAGNOSTIC GROUPS}

Using the operational criteria, we have been able to clinically define alcoholics with Wernicke's encephalopathy neuropathology. Only two institutionalised ( $>15$ years) alcoholics with Korsakoff's psychosis had Wernicke's encephalopathy neuropathology without enough clinical evidence of the disease (false positive rate of $6 \%$ excluding patients with hepatic encephalopathy ). This substantially reduces the current rate $(>80 \%)$ of negative clinical diagnosis, ${ }^{12}$ and the use of the operational criteria prospectively is likely to eliminate this error altogether. The high rate of negative clinical diagnosis of Wernicke's encephalopathy in patients with positive neuropathology in past studies strongly suggests that most patients with clinical Wernicke's encephalopathy go undetected during life, rather than the alternative explanation of 
patients with a clinical episode of Wernicke's encephalopathy having resolving neuropathology.

The question of whether Wernicke's encephalopathy is both clinically and neuropathologically resolvable can be assessed in those alcoholics with neurological symptoms but no neuropathology. In the present study 48 out of the 106 patients examined had no neuropathological abnormality. Most of these $(69 \%)$ also had no signs of Wernicke's encephalopathy in their medical records or from informant interviews with general practitioners or family members. Of the remaining patients (alcohol rated brain dysfunction) most were epileptic from young adulthood or had longstanding neuropsychiatric syndromes. Although it cannot be discounted that some of these neurologically impaired patients may have had a past episode of clinical Wernicke's encephalopathy without any permanent brain damage (true negatives), none had any evidence or history of dietary deficiency, eye signs, or an altered mental state, suggesting that overt encephalopathy is unlikely to have been missed. Mild memory impairment was common in these patients and cerebellar dysfunction was seen in $36 \%$, although coexistent epilepsy and longstanding treatment with anticonvulsants may account for these symptoms. ${ }^{33}$ Therefore, it seems unlikely that a significant number of alcoholics have had Wernicke's encephalopathy clinically without evidence of permanent brain damage.

The finding that Wernicke's encephalopathy and hepatic encephalopathy occur together in some alcoholics has important implications for the acute management and treatment of patients with suspected hepatic encephalopathy. It is well established that the most common and often isolated sign of Wernicke's encephalopathy is a disturbed mental state ${ }^{12}$ and that some patients present in coma ${ }^{34} 35$ making differential diagnosis difficult. The prospective use of the operational criteria outlined should improve diagnostic classification of patients with these life threatening encephalopathies. In particular, previous cerebellar signs or mild memory impairment are more common in Wernicke's encephalopathy than hepatic encephalopathy, with dietary deficiencies and eye signs distinguishing patients with Wernicke's encephalopathy. In addition, alcoholics with both encephalopathies were notable for having physical signs in many domains. Our results indicate that in patients suspected of having alcoholic hepatic encephalopathy in the presence of or with a history of neurological abnormalities, a strong suspicion of Wernicke's encephalopathy should be entertained and appropriate treatment measures taken.

Using the operational criteria descibed, alcoholics with Wernicke's encephalopathy can be distinguished from those with the Wernicke-Korsakoff syndrome with a high degree of sensitivity and specificity. In the present study, two factors distinguished between the diseases: an amnesic syndrome with (1) a clear sensorium and (2) which does not remit with treatment. That is, alcoholics with the Wernicke-Korsakoff syndrome can be differentiated from alcoholics with Wernicke's encephalopathy but without Korsakoff's psychosis by the severity and stability of memory loss regardless of other cognitive deficits. It is important to distinguish permanent memory loss in alcoholics who are sober and thiamine replete from memory loss in intoxicated, thiamine deficient alcoholics. One important test of this distinction may be memory recovery with thiamine supplementation. In agreement with our definition, past studies define the Wernicke-Korsakoff syndrome clinically as an amnesic syndrome in the presence of preserved intelligence and learned behaviour. ${ }^{18-203637}$ In particular, alcoholics with Wernicke-Korsakoff syndrome have a consistent loss of anterograde episodic memory. ${ }^{18} 3638$ whereas many past studies have not emphasised the underlying signs of Wernicke's encephalopathy in patients with the WernickeKorsakoff syndrome, most support the suggestion that the Wernicke-Korsakoff syndrome is the inevitable outcome of Wernicke's encephalopathy. ${ }^{18} 2939$ Interestingly, in our test population this occurred only in the proportion of alcoholics whose survival time was extended by institutional intervention, as previously suggested. ${ }^{40}$ The inclusion of Wernicke's encephalopathy as a prerequisite in our criteria for the Wernicke-Korsakoff syndrome was useful in excluding patients with memory deficits from other causes and did not significantly decrease diagnostic sensitivity. However, the use of Wernicke's encephalopathy as an essential inclusion criterion for Wernicke-Korsakoff syndrome was a source of error for retrospective analysis in a small proportion of patients.

We were unable to identify a subset of alcoholic patients with a dementia-like process when degenerative neuropathology and head injury were excluded. This supports other pathological studies which have not been able to define pathological characteristics for alcoholic dementia. ${ }^{2841}$ The absence of alcoholic dementia in the present sample suggests that coincident neurodegenerative conditions contribute to this clinical profile. Alcoholic dementia has been used to describe those who have a gradual cognitive decline, ${ }^{29}$ whereas the term Korsakoff's psychosis has been used for patients whose onset of memory loss is rapidly acquired. The Wernicke-Korsakoff syndrome is generally diagnosed in younger patients than those with alcoholic dementia. ${ }^{29} 4243$ After excluding degenerative conditions, the alcoholics studied had a mean age (SD) of 58 (10) years and none had gradual cognitive decline. Careful clinicopathological studies have yet to substantiate the existence and aetiology of this proposed condition.

\section{IMPLICATIONS FOR PATHOGENESIS}

The association between the reversible encephalopathic symptoms of Wernicke's encephalopathy and permanent brain damage is not well understood. The neuropathology underlying Wernicke's encephalopathy can 
vary in type (acute $v$ chronic) and extent, ${ }^{23}$ and some of these differences may account for the reversible nature of some symptoms and signs, in particular the eye signs. Lesions in brainstem periventricular regions are likely to account for the eye signs with the type and extent of damage determining their clinical resolution. ${ }^{10}$ By contrast, cerebellar dysfunction is seen as a permanent neurological sign in alcoholics. ${ }^{1410}$ The coexistence of cerebellar abnormalities and poor nutritional state in many patients with Wernicke's encephalopathy supports the suggestion that thiamine deficiency is important in the pathogenesis of cerebellar damage. ${ }^{344}$ Although often termed alcoholic cerebellar degeneration, a direct neurotoxic effect of alcohol has not been shown. Furthermore, malnourished non-alcoholics have also been reported to have cerebellar degeneration. ${ }^{44} 45$ In addition, a proportion of alcoholics with a history of seizures have permanent cerebellar degeneration which may be due to either repeated hypoxic episodes caused by seizures or to longstanding medication with anticonvulsants. ${ }^{33}$

Patients with Wernicke's encephalopathy had a progressive and relentless course with a maximum survival time of three years. By contrast, patients with the Wernicke-Korsakoff syndrome had considerably longer disease durations due to substantial institutionalised care. The importance of rehabilitation in the successful long term management of patients with the Wernicke-Korsakoff syndrome has been noted previously. ${ }^{40} 46$ The increased survival time of institutionalised patients with the Wernicke-Korsakoff syndrome suggests that the brain damage underlying the disorder may be related to cessation of alcohol or correction of thiamine deficiency. Interestingly, in some patients with Wernicke's encephalopathy seizures precede the amnesia necessary for a diagnosis of the Wernicke-Korsakoff syndrome (all patients with the WernickeKorsakoff syndrome with seizures also had Wernicke's encephalopathy neuropathology). This suggests that the Wernicke-Korsakoff syndrome may occur by different pathogenic mechanisms: brain damage in association with severe thiamine deficiency alone (the majority), or the combined brain damage due to thiamine deficiency and seizures.

\section{Summary}

We have devised operational criteria to significantly improve the identification of patients with Wernicke's encephalopathy. The use of these proposed criteria for the differential diagnosis of alcoholics may improve our understanding of the underlying aetiological factors contributing to their neurological impairment. The existing classic triad was modified to include the presence of dietary deficiencies and required only two rather than three signs for a clinical diagnosis. Using this criterion, the diagnosis of Wernicke's encephalopathy either alone or with amnesia (Wernicke-Korsakoff syndrome) or hepatic encephalopathy improved from $22 \%$ to $85 \%$.
Notably, Wernicke's encephalopathy neuropathology was found in a significant number of patients with hepatic encephalopathy, suggesting that these have a high risk of additional Wernicke's encephalopathy and should be treated with parenteral thiamine.

This study was funded by the National Health and Medical Research Council of Australia. We are grateful for the assistance of the staff of the Histopathology Laboratory, Department of Pathology, University of Sydney, and to Professors RF Butterworth and J Willoughby and Dr JDG Watson for their comments on the manuscript.

\section{Appendix}

BRIEF HISTORIES OF REPRESENTATIVE PATIENTS

Alcoholics with hepatic encephalopathy

Patient 1 was a 51 year old male alcoholic who had had the signs and symptoms of hepatic encephalopathy for the preceding two months with two hospital admissions. He initially presented with bleeding oesophageal varices and was discharged one month later when stabilised. He was readmitted one week later drowsy, jaundiced, and dehydrated. For the next three weeks he had recurrent variceal bleeds and resistant ascites with increasing encephalopathy. Six days before death he had a large haematemesis and became hypotensive. $\mathrm{He}$ was resuscitated, but his condition remained grave until death.

Patient 2 was a 65 year old male alcoholic who had had three major hospital admissions over the preceding 15 months for the management of his alcoholic liver disease. He was abstinent for this time period. About one year before death he presented with several days history of weakness, confusion, and disorientation and was admitted to hospital for management of his hepatic encephalopathy. During the next nine months he had further short hospital stays for increased ascites. On final admission he presented semiconscious and hypothermic with evidence of aspirant in the right lung and ascites. He was rousable and responded to name but had low blood pressure, was acidotic, and had ascites with a very high white cell count. Antibiotics were started and CT ordered to discount intracranial haemorrhage; however, on rewarming he deteriorated and died despite intubatation.

\section{Alcoholics with Wernicke's encephalopathy alone}

Patient $3^{27}$ was a 51 year old male alcoholic who had had increasing periods of binge drinking accompanied by poor diet over the preceding three years. Three days before death he was admitted to hospital, heavily intoxicated, with delirium, confusion, and agitation. $\mathrm{He}$ showed ophthalmoplegia, dysarthria, and strabismus tremor. He was given thiamine and became alert and oriented briefly before vomiting large amounts of blood and bile and aspirating.

Patient 4 was a 59 year old male alcoholic who had no major alcohol related diseases besides Wernicke's encephalopathy. When first assessed in 1985 for a noticeable Spigelian hernia, he was able to give a detailed, informative family and personal history. He was immediately admitted to hospital and underwent successful surgery but became confused and disoriented in time and place but not person over the next week and experienced some hallucinations indicative of alcohol withdrawal. $\mathrm{He}$ was discharged one month later. Two years before death he was admitted to hospital for detoxification, showing signs of nystagmus, ophthalmoplegia, and confusion. He was seen at the neuropsychology unit and tested after seven days sobriety. At this time he showed poor planning and construction and scored $26 / 38$ on the short mental status examination despite scoring only $1 / 4$ for simple calculations, $1 / 3$ for similarities, and 0/4 for construction. $\mathrm{He}$ was able to learn four objects after two trials and could remember two of four objects after a five minute delay. Because of defective vision, all memory tests were given 
in the auditory mode. One month before death, he was admitted to hospital during an acute Wernicke's encephalopathy episode with ocular signs, confusion (disoriented in time and place, with no recall of the previous nights events), and severe ataxia. He did not improve with thiamine therapy ( $100 \mathrm{mg}$ daily). Forma testing disclosed global impairment with the examiners concluding that Wernicke's encephalopathy had partially resolved with thiamine therapy. However, reassessment 10 days later showed substantial improvement in attention span, alertness, and ability to follow simple commands. He was discharged three weeks later. The next week he was readmitted with back pain and was again confused and disoriented in time and place. Just before death, his memory skills were poor and he was grossly confused, although the memory lapses were not continuous nor of sufficient degree or nature to be consistent with a diagnosis of Korsakoff's psychosis. This history indicates a persisting confusional state related to heavy ethanol intoxication and thiamine deficiency.

Alcoholics with the Wernicke-Korsakoff syndrome Patient 5 was a 78 year old female alcoholic who had been under constant institutionalised care for eight years before death and had been diagnosed with Korsakoff's psychosis before admission. Estimates of alcohol intake were in excess of $100 \mathrm{~g}$ per day from the age of 20 without adequate diet. The first report of memory impairment was 20 years before death. She was persistently disoriented in time and place and had nystagmus, cerebellar ataxia, and severely impaired declarative memory. She was unable to remember her doctor from week to week despite being in his care for six years. Disorientation and amnesia persisted until death despite documentation of abstinence from alcohol for the final eight years. This neuropsychological profile indicates the persisting, stable memory impairment diagnostic of Korsakoff's psychosis.

Patient 6 was a 60 year old chronic alcoholic who was admitted to hospital in 1980 for severe headaches, when it was noted that he had problems with his memory. In 1983, he was admitted to hospital with chest pains and during investigations was noted to have nystagmus and ataxia. His memory, however, was regarded as normal. He was readmitted to hospital in 1990 because of suicidal intention with bilateral nystagmus and a broad based ataxic gait. Neuropsychologica testing showed him to be a man of average to above average intelligence, with a normal immediate memory span (short mental state 30/38). However, he was unable to learn any new material (structured or unstructured) beyond his immediate memory span. He recalled none of four words after five minutes, and there was nearly total loss of information after interference. $\mathrm{He}$ was unable to recall personal events from the previous 10 years. He was institutionalised without improvement in memory function for almost three years until his death by suicide. This neuropsychological profile indicates the persisting, stable memory impairment of Korsakoff's psychosis.

\section{Alcoholics with alcohol related brain dysfunction}

Patient 7 was a 58 year old male alcoholic who developed epilepsy a year before death with no CT abnormality. Three months before death he was diagnosed with carcinoma of the lung and declined treatment. He had several grand mal seizures in the two days before death.

Patient 8 was a 66 year old male alcoholic who haddrank heavily since the age of 18 . Examination in the neuropsychological unit disclosed a mild memory deficit. He was able to learn four unrelated words after one attempt, but his recall was very poor. It was not until he had repeated the test that he was able to remember two of the four words. All other tests were adequate. Peripheral neuropathy was noted.

Alcoholics without neurological complications

Patient 9 was a 41 year old man with a long history of alcohol misuse. By the age of 29 he had had 34 admis- sions to detoxification units. On neuropsychological assessment 18 months before death no impairment was noted. He scored $37 / 38$ on the short mental state examination and had insight into his alcohol problem. He remained cognitively stable until death, which was due to acute alcohol intoxication.

\section{Alcoholics with multiple diagnoses}

Patient 10 was a 46 year old woman who presented with hepatic encephalopathy after a 27 year history of consuming about one bottle of scotch a day. Two and a half years before death she was admitted for one month after a short history of weight loss, vomiting, anorexia, and increasing jaundice with hepatomegaly and raised serum transaminases. Three months before death, she was admitted with hepatomegaly and jaundice, but no evidence of oesophageal varices or peptic ulceration. Peripheral neuropathy and cerebellar dysfunction were evident. Clinical evidence of encephalopathy returned after reduction of dietary protein content. She had a short history of auditory and visual hallucinations. She had asterixis, spider naevae, pronounced jaundice, clubbing of fingers and toes, and hepatosplenomegaly. Investigations disclosed anaemia, hyponatraemia, high serum, and urinary osmolarity, and pulmonary oedema. She was assessed by the neuropsychology unit denying any symptoms of cognitive impairment or neurological dysfunction. Bilateral nystagmus was present on lateral gaze and there was generalised muscle wasting. She had an abnormal tandem gait and abnorma heel-shin test although she was not clinically encephalopathic. On short mental state examination she scored 20 out of 38 with problems in digit span, learning, calculations, information, similarities, construction, and recall. On full neuropsychological assessment she showed severe memory impairment and pronounced impairment of higher order cognitive functions. Confusion developed and hepatic failure with malaena became evident, although renal failure supervened.

Case 11 was a 46 year old male alcoholic with a 10 year history of alcohol-related diseases including bleeding gastric ulcers, chronic liver disease, nystagmus, tremor, memory blanks and a five year history of ataxia and frequent vomiting. $\mathrm{He}$ was first noted to have memory problems in early 1990 when he returned to his doctor's surgery having forgotten his prior attendance that morning. In the final year of his life, he was still living independently although he was often disoriented in time and place and showed gross memory loss and unsteady gait. $\mathrm{He}$ was admitted to hospital with epigastric pain one month before death, was confused, disoriented in time and place, and had poor short term memory. His family reported an increase in mental impairment over the previous fortnight. He was formally tested two weeks later and was found to have a general amnesic syndrome. Although alert and cooperative, he showed perserveration and confabulation. He could do no calculations and could not remember facts from personal or historical events. He was last admitted to hospital in hepatic coma, surviving for three days. This history indicates an amnesic deficit of at least 12 months duration with intermittent episodes of Wernicke's encephalopathy related to heavy ethanol intoxication and thiamine deficiency followed by a rapidly decompensating liver disease.

1 Harper CG, Giles M, Finlay-Jones R. Clinical signs in the Wernicke-Korsakoff complex: a retrospective analysis of 131 patients diagnosed at necropsy. $\mathcal{f}$ Neurol Neurosurg Psychiatry 1986;49:341-5.

2 Naidoo DP, Bramdev A, Cooper K. Wernicke's encephalopathy and alcohol-related disease. Postgrad Med encephalopathy and

3 Kril J. Neuropathology of thiamine deficiency disorders. Metab Brain Dis 1996;11:9-17.

4 Watson JDG, Kril JJ, Lennane KJ. Neurological, neuropathological and psychiatric aspects of alcoholism Medicine International 1995;23:61-5.

5 Shogry MEC, Curnes JT. Mamillary body enhancement on MR as the only sign of acute Wernicke encephalopathy. Am $\mathcal{F}$ Neuroradiol 1994;15:172-4.

6 Vortmeyer AO. Wernicke's encephalopathy, Korsakoff's amnesic state and thiamine deficient encephalopathy. Alcohol 1993;28:199-200. 
7 Butterworth RF. Pathophysiologic mechanisms responsible for the reversible (thiamine-responsive) and irreversible (thiamine non-responsive) neurological symptoms of Wernicke's encephalopathy. Drug Alcohol Rev 1993;12: 315-22.

8 Charness ME. Brain lesions in alcoholics. Alcohol Clin Exp Res 1993;17:2-11.

9 Price J, Kerr R. The Wernicke-Korsakoff syndrome: clinical corelates and dilemmas. Aust Drug Alcohol Rev 1988 7:57-60.

10 Victor M, Adams RD, Collins GH. The Wernicke-Korsakoff Syndrome. 2nd ed. Philadelphia: FA Davis Company, 1989

11 D'Aprile P, Gentile MA, Carella A. Enhanced MR in the acute phase of Wernicke encephalopathy. $A m$ F Neuroradiol 1994;15:591-3.

12 Arriagada PV, Marzloff K, Hyman BT. Distribution of Alzheimer-type pathologic changes in non-demented elderly individuals matches the pattern in Alzheimer's disease. Neurology 1992;42:1681-8.

13 McKhann G, Drachman D, Folstein M, Katzman R, Price D, Stadlan EM. Clinical diagnosis of Alzheimer's disease: Report of the NINCDS-ADRDA work group under the auspices of the Department of Health and Human the auspices of the Department of Health and Human
Services task-force on Alzheimer's disease. Neurology Services task-force
1984;34:939-44.

14 Morris JC, Heyman A, Mohs RC, et al. The consortium to establish a registry for Alzheimer's disease (CERAD). I. Clinical and neuropsychological assessment of Alzheimer's disease. Neurology 1989;39:1159-65.

15 Harper C, Fornes P, Duyckaerts C, Lecomte D, Hauw JJ. An international perspective on the prevalence of the Wernicke-Korsakoff syndrome. Metab Brain Dis 1995; 10:17-24.

16 Darnton-Hill I, Truswell AS. Thiamin status of a sample of homeless clinic attenders in Sydney. Med 7 Aust 1990; 152:5-9.

17 Butterworth RF. Hepatic encephalopathy. The Neurologist 1995;1:95-104.

18 Butters N, Stuss DT. Diencephalic amnesia. In: Boller F, Grafman J, eds. Handbook of neuropsychology. Elsevier Grafman J, eds. Handbook of neurops

19 Kopelman MD. The Korsakoff syndrome. $B r f$ Psychiatry 1995;166:154-73.

20 O'Connor M, Verfaellie M, Cermak LS. Clinical differentiation of amnesic subtypes. In: Baddeley AD, Wilson BA, Watts FN, eds. Handbook of memory disorders. New York: John Wiley and Sons, 1995;53-80.

21 Spreen O, Strauss E. A compendium of neuropsychological tests. Oxford: Oxford University Press, 1991.

22 Harper C. Wernicke's encephalopathy: a more common disease than realised. $\mathcal{f}$ Neurol Neurosurg Psychiatry 1979; 42:226-31.

23 Torvik A. Topographic distribution and severity of brain lesions in Wernicke's encephalopathy. Clin Neuropathol 1987;6:25-9.

24 Strub RL, Black FW. The mental status examination in neurology. 2nd ed. Philadelphia: FA Davis Co, 1985.

25 Rice JP, Reich T, Bucholz KK, et al. Comparison of direct interview and family history diagnosis of alcohol dependence. Alcohol Clin Exp Res 1995;19:1018-23.

26 McKeith IG, Fairbairn AF, Perry RH, Thompson P. The clinical diagnosis and misdiagnosis of senile dementia of
Lewy body type (SDLT). Br $\mathcal{F}$ Psychiatry 1994;165: 324-32.

27 Byrne C, Halliday G, Ellis J, Harper C. Thalamic vacuolation in acute Wernicke's encephalopathy. Metab Brain Dis 1993;8:107-14.

28 Victor M. Alcoholic dementia. Can f Neurol Sci 1994;21: 88-99.

29 Cutting J. The relationship between Korsakov's syndrome and "alcoholic dementia". $B r f$ Psychiatry 1978;132: 240-51.

30 Butters $N$. Alcoholic Korsakoff's syndrome: some unresolved issues concerning etiology, neuropathology, and cognitive deficits. $\mathcal{f}$ Clin Exp Neuropsychol 1985;7: 181-210.

31 Bowden SC. Separating cognitive impairment in neurologically asymptomatic alcoholism from Wernicke-Korsakoff Syndrome: is the neuropsychological distinction justified? Psychol Bull 1990;107:355-66.

32 Waugh $M$, Jackson M, Fox GA, Hawke SH, Tuck RR. Effects of social drinking on neuropsychological performance. Br $f$ Addict 1989;84:659-67.

33 Sobaniec-Lotowska ME, Sobaniec W. Morphological features of encephalopathy after chronic administration of tures of encephalopathy after chronic administration of
the antiepileptic drug valproate to rats. A transmission the antiepileptic drug valproate to rats. A transmission
electron microscopic study of capillaries in the cerebellar electron microscopic study of capillaries

cortex. Exp Toxic Pathol 1996;48:65-75.
34 Wallis WE, Willoughby E, Baker P. Coma in the WernickeKorsakoff syndrome. Lancet 1978;2:400-1.

35 Torvik A, Lindboe CF, Rogde S. Brain lesions in alcoholics. A neuropathological study with clinical correlations. F Neurol Sci 1982;56:223-48.

36 Squire LR, Knowlton B, Musen G. The structure and organization of memory. Ann Rev Psychol 1993;44:453-95.

37 Zola-Morgan S, Squire LR. Neuroanatomy of memory. Ann Rev Neurosci 1993;16:547-63.

38 Moss MB, Albert MS, Butters N, Payne M. Differential patterns of memory loss among patients with Alzheimer's disease, Huntington's disease, and alcoholic Korsakoff's syndrome. Arch Neurol 1986;43:239-46.

39 Ryan C, Butters N. Further evidence for a continuum-ofimpairment encompassing male alcoholic Korsakoff patients and chronic alcoholic men. Alcohol Clin Exp Res 1980;4:190-8.

40 Price J, Kerr R, Hicks M, Nixon PF. The WernickeKorsakoff syndrome: a reappraisal in Queensland with special reference to prevention. Med f Aust 1987;147: $561-5$.

41 Joyce EM. Aetiology of alcoholic brain damage: alcoholic neurotoxicity or thiamine malnutrition? Br Med Bull 1994;50:99-114.

42 Carlen PL, McAndrews MP, Weiss RT, et al. Alcoholrelated dementia in the institutionalized elderly. Alcohol Clin Exp Res 1994;18:1330-4.

43 Whelan G. Alcohol-related health problems in the elderly. Med f Aust 1995;162:325-7.

44 Adams RD. Nutritional cerebellar degeneration. Amsterdam: Elsevier, 1976.

45 Mancall EL, McEntee WJ. Alterations of the cerebellar cortex in nutritional encephalopathy. Neurology 1965;15: 303-13.

46 Lennane KJ. Management of moderate to severe alcoholrelated brain damage (Korsakoff's syndrome). Med $\mathcal{F}$ Aust 1986;145:136-43. 